\section{$\underset{\substack{\text { hommes } \\ \text { \& migrations }}}{ }$}

Hommes \& migrations

Revue française de référence sur les dynamiques

migratoires

1302 | 2013

Le Japon, pays d'immigration?

\title{
Esquisse d'une approche comparative des situations migratoires au Japon et en France
}

Abdelhafid Hammouche

\section{(apenEdition}

\section{Journals}

\section{Édition électronique}

URL : http://journals.openedition.org/hommesmigrations/2457

DOI : 10.4000/hommesmigrations.2457

ISSN : 2262-3353

\section{Éditeur}

Musée national de l'histoire de l'immigration

\section{Édition imprimée}

Date de publication : 1 avril 2013

Pagination : 13-20

ISBN : 978-2-919040-22-3

ISSN : $1142-852 X$

\section{Référence électronique}

Abdelhafid Hammouche, «Esquisse d'une approche comparative des situations migratoires au Japon et en France », Hommes \& migrations [En ligne], 1302 | 2013, mis en ligne le 31 décembre 2015, consulté le 21 avril 2019. URL : http://journals.openedition.org/hommesmigrations/2457 ; DOI : 10.4000/hommesmigrations.2457 


\title{
ESQUISSE D'UNE APPROCHE COMPARATIVE DES SITUATIONS MIGRATOIRES
} AU JAPON ET EN FRANCE

par ABDELHAFID HAMMOUCHE, professeur des universités, Lille-I, directeur du Clersé CNRS (UMR 8019 USTL-CNRS)

\author{
La France et I'Algérie, le Japon et le Brésil, voilà deux couples \\ migratoires propices à la comparaison. Sans faire d'analogies \\ entre la structuration de leurs situations migratoires respectives, \\ il s'agit de faire place, dans l'analyse, au double regard \\ permettant de saisir à la fois les réalités de la société d'accueil \\ et celles de la société d'origine. Le retour des Nippo-Brésiliens \\ au Japon, puis leur départ à nouveau vers le Brésil n'est \\ pas sans rappeler les parcours des Algériens entre la France \\ et I'Algérie.
}

Comprendre les relations migratoires au Japon

La France est fréquemment présentée comme une terre d'accueil, avec la flatteuse référence d'être celle qui a donné naissance aux droits de l'homme. Le Japon n'équivaut pas, dans les représentations les plus répandues, à la figure inverse, mais néanmoins à celle d'un archipel plutôt fermé sur lui-même. Cet article propose d'écarter un tel clivage par une amorce d'approche comparative en contextualisant les migrations dans les deux pays. Approche comparative ne signifie pas comparaison terme à terme, mais essayer de comprendre et de mettre en parallèle, ou en relation, ce qui apparemment n'est pas toujours comparable, pour entretenir une constante attention à l'environnement anthropologique au sein duquel prennent sens les mouvements et les situations migratoires. La déstabilisation escomptée du regard devrait engendrer une interrogation sur nos propres notions, découpages statistiques et catégories de perception. On essaiera de comprendre ce qui caractérise une partie des relations migratoires au Japon, en esquissant un parallèle avec la situation française.

À défaut d'une histoire sociale des migrations concernant ce pays, dont on trouvera des aperçus dans les différentes contributions de ce numéro, on rappellera quelques éléments caractéristiques de l'émigration japonaise, avant de nous intéresser principalement à la relation entre le Japon et 
le Brésil, et secondairement à la relation entre la France et l'Algérie. Il eût été plus pertinent d'établir un parallèle avec la relation Japon-Corée à laquelle songent la plupart des observateurs de la société japonaise, comme

Le pays a connu différentes

vagues migratoires dans

son histoire. Globalement,

et selon l'estimation de 2004 ,

on compte près de 2,6 millions

d'émigrés japonais dans

le monde, dont 1,5 million

en Amérique du Sud. une sorte d'équivalent de la relation franco-algérienne ${ }^{1}$. Mais, faute de travaux ${ }^{2}$ et compte tenu des recherches de l'auteur de ces lignes, le choix s'est fait sur la relation Japon-Brésil qui, sous l'angle des renversements de perspectives pour les migrants, offre de riches enseignements. Nous fonderons notre propos sur les recherches dont rendent compte les Cahiers du Brésil contemporain ${ }^{3}$ consacrés au centenaire de l'immigration japonaise au Brésil, sur le numéro d'Hommes \& Migrations portant sur les migrations au Brésil ${ }^{4}$, ainsi que sur les contributions du présent numéro.

\section{Penser les situations migratoires par une double contextualisation}

Pour écarter les stéréotypes d'un archipel fermé sur lui-même, ou générant une émigration seulement orientée sur l'appropriation des technologies des pays européens, comme on l'a vu au XIX siècle (ère Meiji), il faut souligner que le pays a connu différentes vagues migratoires dans son histoire. Globalement, et selon l'estimation de 2004, on compte près de 2,6 millions d'émigrés japonais dans le monde, dont 1,5 million en Amérique du Sud 5 . Il convient aussi de rappeler la relation particulière entre le Japon et les États-Unis, où les rapports économiques et politiques étroits, et pour partie hérités de la Seconde Guerre mondiale, se combinent avec la présence d'une communauté de Japonais qui a connu de sensibles changements de statut. Les Nippo-Américains étaient en effet au nombre de 122177 en 2007 en incluant ceux qui se déclarent "d'origines mixtes". Ils se répartissent pour le plus grand nombre entre la Californie (394 896), Hawaï (296 674), l’État de Washinton (56 210), New York (45 237). Près de 7000 Japonais entrent aux États-Unis chaque année alors que des personnes âgées retournent au Japon. Cette émigration, qui a commencé au milieu du XIX siècle, a connu de lourdes discriminations, depuis la ségrégation organisée en 1893 pour cantonner la scolarité des enfants japonais avec celle des jeunes Chinois, l'émergence de l'idée du "péril jaune" à San Francisco en 1905, le jugement du 13 novembre 1922 par lequel la Cour suprême interdit aux immigrants japonais d'acquérir la nationalité américaine, jugement qui ne sera aboli qu'en 1952, jusqu'à l'enfermement dans des camps lors de la Seconde Guerre, ce qu'ont dévoilé de récents écrits ${ }^{6}$.

Parmi les migrations, celle à destination du Brésil est aussi particulière à la fin du XX $X^{\text {e }}$ siècle, avec une tentative de "retour". Il est vrai que d'autres émigrés au Pérou, ou en Argentine, connurent le même sort, mais en moins grand nombre. L'arrivée des Japonais au Brésil débute dans les années 1900. Ceux-ci ne formaient pas, comme le précise Ruth Corrêa Leite Cardoso, une unitép. Il y a eu ceux qui ont débarqué dans la forêt amazonienne où ils ont cultivé le jute, puis d'autres agriculteurs originaires de l'archipel se sont installés dans le nord du Paraná ou dans des régions rurales ou urbaines, changeant parfois de métier pour se tourner vers le commerce ou l'industrie. 
Madame Kim s'est mariée en 1992 avec un Coréen. Avant de tenir ensemble une boutique d'électroménager dans le quartier de Shin-Okubo, ils étaient commerçants à Bamako où ils ont connu M. Diarra.

(c) Camille Millerand

Certains États, comme celui de Sao Paulo ${ }^{8}$, le plus gros producteur de café du pays, ont accueilli quelque 250000 travailleurs (hommes et femmes) en un flux continu entre 1908 et 1970 (sauf entre 1942 et 1945, pour cause de guerre) ${ }^{9}$.

Dans les premiers temps, jusqu'à la Seconde Guerre mondiale, les arrivées successives de Japonais au Brésil concernent une émigration familiale générée par les contrats binationaux.

La reprise de l'émigration dans les années 1950 implique cette fois-ci des jeunes gens célibataires, que les migrants déjà installés désignent par l'expression "le nouveau Japon". C'est ensuite la vague des "retours" dans l'archipel qui débute dans les années 1980. Les flux augmentent, comme le soulignent Naoto Higuchi et Nanako Inaba, jusqu'en 2007, avant de reculer d'un tiers en 2011. Près de 30 \% de ces Nippo-Brésiliens vont conclure l'expérience par un nouveau départ à destination du Brésil.

Exception faite du rapatriement des colons d'Algérie en 1962, qui résulte du contexte particulier de la décolonisation, il n'y eut pas de phénomène équivalent en France. Même en élargissant pour prendre en compte les retours des immigrés depuis la France, par exemple en comptabilisant les "aides au retour" du dispositif mis en place par Lionel Stoleru en 1977, on n'atteint pas l'ampleur durant ce dernier demi-siècle de la vague de réinstallation au pays d'origine comme celle des dekasseguis au Japon. Mais, pour ne pas laisser penser qu'il s'agit là d'une exception confirmant que les migrations se soldent seulement par une sédentarisation, il est intéressant d'observer que certains migrants algériens installés de longue date en France contribuent à leur manière aussi à diversifier les rapports à leurs deux sociétés de référence et instaurent une double installation (par exemple des retraités qui vont et viennent), écartant ainsi la seule perspective clivée du retour ou de l'installation.

\section{Le métissage en question}

Cette dynamique impliquant les deux pays concernés par le parcours migratoire, différemment selon les périodes historiques, tour à tour société d'accueil et société d'origine, incite à considérer simultanément les deux volets émigration-immigration. La double contextualisation opérée de la sorte fait ressortir non seulement la complexité des mouvements de peuplement, en relativisant la seule perspective de la sédentarisation, et met encore plus en relief les appréciations conjoncturelles des métissages. Comme le montre Frédéric Roustan dans ce numéro, le métissage est valorisé au Japon pour les uns, lorsque la volonté de "rattraper" les Européens l'emporte, et "dévalorisé", lorsque 
l'expansion impériale coloniale apparaît aux yeux de certains comme un risque de dilution de la domination ethnico-culturelle. Il en va de même pour la France où, dans les années 1950, le docteur Marchand ${ }^{10}$ désignait les couples franco-algériens comme des monstruosités, alors que dans les années 1990, ils étaient présentés comme des indicateurs d'intégration ${ }^{11}$.

Il est vrai qu'avec ces exemples ce ne sont pas les mêmes catégories d'acteurs qui se prononcent dans les deux pays. Dans le contexte japonais, c'est le personnel politique et administratif, et plus largement les cercles du pouvoir, qui énoncent les appréciations sur les "mélanges" profitables ou non selon la perspective expansionniste ou défensive qu'ils adoptent. Dans le contexte français, l'appréciation s'entend d'abord dans une configuration coloniale avec un ex-pays colonisé et met en avant un notable algérois de la fin de la colonisation française qui dit sa révulsion pour les unions entre Français et Algériens, puis plusieurs décennies plus tard, en métropole, une chercheuse participant aux débats
Au Japon comme en France, les mises à distance s'affirment autant par des stigmatisations symboliques que par des réalités pénalisantes,

I'habitat et plus largement la participation à la vie sociale notamment en ce qui concerne sur la place des enfants d'immigrés dans la France des années 1990. Par contre, la différence entre les systèmes socio-politiques contemporains des deux pays est nette: si la perspective de "l'intégration républicaine" française est controversée ou au moins débattue dans sa mise en œuvre, elle recueille un assez large consensus pour la définition d'une citoyenneté affranchie des empreintes ethniques et ouverte à tous, et par la volonté affichée de combattre les discriminations, alors que la conception japonaise de l'identité demeure, dans bien des propos, attachée à une "origine" protégée par l'insularité.

Il est instructif d'élargir et d'inclure le Brésil dans ce jeu d'appréciation des métissages. Dans ce pays, la miscégénation (pour reprendre ce concept de Roger Bastide $^{12}$ avec lequel il approche les contacts et les relations interculturels) est valorisée, alors qu'elle suscite du scepticisme quant à la réalité de sa traduction politique et sociale. Car, on le sait bien, la promotion discursive ("le Brésil des métissages") ne gomme pas, loin s'en faut, toutes sortes de discriminations qui structurent la société brésilienne. Ainsi, les Nippo-Brésiliens ont fait l'objet d'une désignation durable de "jaunes", qui indique en creux une extériorité persistante à la société brésilienne.

$\mathrm{Au}$ Japon comme en France, les mises à distance s'affirment autant par des stigmatisations symboliques que par des réalités pénalisantes, notamment en ce qui concerne l'habitat et plus largement la participation à la vie sociale, comme on le verra plus loin. Bien sûr, le jugement moral et politique ou l'appréciation méthodique nourrie par des recherches pèsent sur la vie des migrants et de ceux qui leur sont liés. Mais les difficultés sociales, souvent liées aux discriminations, restent les plus déterminantes. D'une part, l'accès à l'emploi demeure le point le plus sensible et la situation plus précarisée des immigrés au Japon n'est pas sans similitudes avec ce qui se passe en France. D'autre part, sur le plan du droit, et à la différence de la législation française qui tendait à faciliter l'acquisition de la nationalité pour le conjoint étranger, les obstacles sont nombreux au Japon, comme on le voit avec les ambiguiités qui frappent le statut de celles qui s'unissent à des immigrés. Dans ce cas, l'épouse japonaise a été longtemps niée, et c'est bien tardivement qu'elle s'est vue reconnaître des droits pour ses enfants. Ce sont là des indices non seulement des modifications du regard porté sur l'étranger et la question de l'identité nationale, mais aussi des changements relatifs aux rapports de genre.

\section{Distinguer les phases migratoires}

Sur le plan scientifique, les désignations ou les conceptualisations gagnent également à être mises en perspective par cette double contextualisation. Les termes pour désigner les immigrés, comme 
"oldcomers" ou "newcomers", pour s'en tenir aux mots anglais utilisés dans les sciences sociales au Japon, distinguent des vagues d'arrivée. Cette catégorisation différencie aussi, pour un grand nombre de ceux qui la reprennent, les anciens sujets de l'empire et les migrants du reste du monde. En effet, les immigrés arrivés avant 1945 venaient pour la plupart de Taiwan et de Corée, alors que ceux qui arrivent à partir des années 1970 sont principalement des Chinois, des Brésiliens, des Philippins et toujours des Coréens. Ces nouveaux migrants entrent pour certains avec un statut d'étudiant (ou comme stagiaires pour trois ans dans les secteurs agricole et industriel), comme c'est le cas pour de nombreux Chinois et Coréens qui parviennent ensuite à accéder à des emplois qualifiés. Les Brésiliens bénéficient d'un visa s'ils sont descendants de Japonais et sont plutôt recrutés dans le secteur industriel. Quant aux Philippins, ils se caractérisent par une plus forte proportion de femmes et par un visa pour des emplois dans le secteur du spectacle, et plus récemment dans celui de la santé. Pour les émigrés, outre le terme générique "nikkei" pour désigner les migrants japonais dans le monde, les migrants aux États-Unis se voient accoler la classification de "issei", d'après la numérotation japonaise, pour la première génération, "nisei" pour la deuxième, et "sansei" pour la troisième. Cette déclinaison générationnelle existe en France où elle est autant banalisée dans le langage courant que contestée lorsqu'elle est reçue comme une assignation d'altérité pénalisante. Cette ambivalence frappant les désignations repose la question souvent entendue ces dernières décennies en France : étrangers ou immigrés aux yeux des autres, mais jusqu'à quand?

Dans l'Hexagone, l'insistance pour différencier les flux porte sans doute moins sur les phases économiques et la nationalité du migrant que sur une distinction entre migrations provenant d'Europe et celles venant de l'ex-empire à la période coloniale ou postcoloniale. À défaut d'un inventaire exhaustif impossible ici, les concepts les plus marquants dans les travaux en sciences sociales en France portent bien plus sur les liens avec le pays d'origine et le pays d'accueil. On pense évidemment à Abdelmalek Sayad qui a le plus clairement exprimé ce lien avec son concept d'émigration-immigration qui exclut de penser un des deux volets indépendamment de l'autre, si on ne veut pas biaiser lourdement l'appréciation et la complexité du phénomène migratoire.

À certains égards, les données et les analyses relatives
Cette ambivalence frappant les désignations repose la question souvent entendue ces dernières décennies en France : étrangers ou immigrés aux yeux des autres, mais jusqu'à quand? gration-immigration nippo-brésilienne élargissent la typologie à laquelle bon nombre de travaux se réfèrent en France, lorsqu'il s'agit de l'émigrationimmigration algérienne. Ces deux "couples" migratoires (France-Algérie, Japon-Brésil) apparaissent différemment dans leur structuration. Ainsi, la typologie des trois âges de Sayad ponctue une décomposition de la société rurale avec une émigration d'abord aux ordres du lignage et à son service (premier âge), puis celle-ci consacre le détachement de la condition paysanne et l'ébranlement de l'indivision au principe de la famille étendue (deuxième âge), alors que la vague suivante traduit le résultat de ce long processus d'individuation prenant sens avec une communauté d'immigrés plus conséquente (troisième âge) ${ }^{13}$.

Les relations entre les générations, la recomposition des rapports à la culture d'origine et à la société d'accueil se reflètent dans l'énonciation des classes d'âge ou de génération (la "première", la "deuxième", la "troisième" génération, ou les issei, les nisei et les sansei), mais au risque de laisser entendre la seule dilution des traits culturels des primo-migrants et le détachement pour les suivants. Les travaux récents écartent cette réduction à la seule perspective d'assimilation ou d'intégration, pour se focaliser davantage sur la dynamique culturelle tant dans le pays d'origine que dans le pays d'accueil, pour mieux montrer des héritages 
Ibrahim vient de trouver un nouveau job en tant qu'ouvrier spécialisé dans une imprimerie située à une heure de transport de chez lui. Pour sa retraite, il rêve de s'acheter un terrain au Mali et de quitter définitivement l'archipel. (c) CAmille Millerand

pratiques et symboliques et des appropriations plus complexes ${ }^{14}$. Pour étoffer l'analyse en ce sens, il serait sans doute éclairant d'inclure un autre registre des désignations, celui des autodéfinitions produites par les migrants et leurs enfants pour se définir eux-mêmes, et qui constituent autant d'appréciations de la situation migratoire. On l'entend par exemple avec le mot "khoroto", largement utilisé en France et en Algérie par les primo-migrants algériens dans les années 1970-1980 et qui signale autant un écart de langage que l'indication d'une conduite limite. Ce terme, selon les âges et les parcours, exprime une complicité entre locuteurs avertis ou signifie une attitude dévalorisée. Il contient éventuellement une autodérision. D'autres termes - comme "beur", le plus médiatisé à partir des années 1980 avec la Marche qui porte ce nom ${ }^{15}$ se forgent par une inversion ("arabe" qui devient "beur"), et sont autant d'indices conjoncturels sur les rapports d'altérité entre proches ou avec les autres supposés plus lointains.

\section{Les débats et la fonction du local}

Si, sur le registre politique, l'histoire coloniale et les procédures administratives pour encadrer les séjours conditionnent de part et d'autre l'implantation et les situations migratoires, il en va différemment pour la structuration de l'action publique dans les deux pays. En France, les politiques dites d'“intégration" ou celles qui se définissent de manière plus large comme "politique de la ville", révèlent une manière de concevoir institutionnellement 
l'accompagnement des migrants et de leurs enfants. Souvent critiquée pour la spatialisation combinée à l'ethnicisation qu'elle génère avec les définitions de périmètre de l'action, cette politique publique est impulsée par les ministères et par les collectivités territoriales, et formellement conçue pour une mise en œuvre impliquant le monde associatif.

Il n'existe pas d'équivalent ni une telle visibilité institutionnelle nationale au Japon. L'action en direction des populations immigrées repose principalement sur les ONG. Et si on suit Takashi Miyagima ${ }^{16}$, la politique à l'endroit des migrants n'est pas assumée délibérément au niveau national. Il en veut pour preuve l'absence de loi spécifique contre le racisme au Japon, et il insiste sur la dimension uniquement locale de l'action qui se conçoit et se réalise avec très peu de moyens. C'est ce qu'indique également Nakano Yûji dans ce numéro, en rappelant que le dernier rapport national consacré à ces questions en 2006 a confirmé que les mesures dédiées à l'intégration reposaient sur l'action des collectivités territoriales. La fonction, de fait, de l'espace local, lorsque celui-ci se voit attribuer, comme en France avec la politique de la ville, une vocation d'expression et de recyclage des appartenances culturelles, semble différente au Japon, où la gestion révèle d'abord une volonté de discrétion et de non-reconnaissance à l'échelon national de la question socio-culturelle. Espace quasi exclusif de l'action dans ce pays, ce dernier est pensé en France en termes d'articulation des territoires et des échelles à partir des services de l'État. Pourtant, sur plusieurs points - les processus de peuplement depuis les années 1970 dans les grands ensembles en France, la place ou la mobilisation escomptée des associations -, le parallèle est tentant.

Certes, les difficultés relatives à la "captivité" résidentielle ne se révèlent ni tout à fait dans les mêmes périodes, ni avec la même ampleur. Cependant, on observe un processus d'appauvrissement des populations et la fonction de cet habitat se transforme au mieux en lieu de transition pour ceux qui possèdent les ressources sociologiques et économiques pour les quitter, et en impasse sociale pour les plus fragiles. Cette dynamique sociale et urbaine donne une plus grande ampleur à ce que signalaient déjà les auteurs de "Proximité spatiale et distance sociale ${ }^{17 "}$ au début des grands ensembles. Il en va de même pour la stigmatisation qui frappe l'accès à l'emploi (l'adresse donnée sur son CV peut être stigmatisante).

\section{De nouveaux points de comparaison}

Il serait sans doute éclairant d'élargir la question pour interroger les stratégies scolaires des familles afin de vérifier si au Japon, où nombre d'enfants d'étrangers ne sont pas ou peu scolarisés, on constate les jeux d'évitement proches de ceux observés en France. Si, de part et d'autre, le poids particulier du chômage et sa structuration spécifique, avec le rapport à la langue, les qualifications et les discriminations quiconditionnentl'accèsàl'emploietla mobilité professionnelle, sont mis en question, il en va autrement pour l'incitation ou le soutien apporté à la participation à la vie politique locale. Outre les politiques dites d'intégration" déjà signalées pour la France, la comparaison met en reliefles nombreux travaux des organismes nationaux comme La double contextualisation esquissée ici s'offre comme une opportunité pour analyser la bipolarité pays d'originepays d'accueil et la situation migratoire, sans s'enfermer dans les schèmes propres à une dynamique nationale. l'Institut national de la statistique et des études économiques (Insee) ou l'Institut national d'études démographiques (Ined), sans compter les recherches menées par divers laboratoires et équipes du Centre national de la recherche scientifique (CNRS), pour alimenter les débats sur la scène publique, comme ceux qui ont porté sur l'appréciation sociodémographique des Français issus de l'immigration ${ }^{18}$. Le débat semble moins 
polémique au Japon sur le poids démographique des étrangers. Il s'exprime par des expressions telle que "cité à forte population étrangère", que rappelle Chikako Mori, et dont la définition est floue tant par l'usage qu'en font aussi bien les chercheurs que le personnel politique ou médiatique. Sur le plan géographique, il conviendrait sans doute d'affiner la comparaison pour aller au-delà du constat que, dans les deux pays, les villes industrielles accueillent le plus grand nombre de migrants. Il serait judicieux d'apprécier la constitution des réseaux d'immigrés propres à certaines régions d'origine, comme il y eut en France des correspondances entre certaines parties du nord de l'Algérie et les grandes métropoles industrielles de la région Rhône-Alpes, par exemple. Les logiques d'attribution de logements, notamment les critères économiques et linguistiques, contribuent à des regroupements apparemment communautaires également au Japon, mais sans que l'on puisse mesurer les liens avec les pays d'origine. Il ne s'agit pas pour autant de "s'enfermer" dans le local, mais bien d'approcher les situations et les configurations relationnelles pour mieux mettre en question la "place" des uns et donc aussi celle des autres, pour des enjeux aussi déterminants que l'accès à l'emploi, ou la considération sociale et symbolique des minorités, de la légitimité à être "là", sans dévaluation dans la vie sociale au sens large.

\section{Conclusion}

La double contextualisation esquissée ici s'offre comme une opportunité pour analyser la bipolarité pays d'origine-pays d'accueil et la situation migratoire, sans s'enfermer dans les schèmes propres à une dynamique nationale. On pourrait dans cette perspective prolonger le questionnement pour évoquer, par exemple, les relations parfois tendues entre migrants récents (ou les positions relatives à la situation des clandestins) et populations plus anciennement installées, quand l'aspect physique ou "l'enracinement" ne peuvent être mobilisés comme arguments aussi fréquemment que lors des migrations les plus étudiées comme celles des Polonais ou des Italiens aux États-Unis, ou des Maghrébins en Europe.

Concernant le Japon et la France, si une partie des populations immigrées est confrontée à des difficultés sociales renforcées par l'ethnicisation des rapports, les actions pour les soutenir ou pour redéfinir les problèmes s'affirment d'abord dans l'espace local. Pourtant, les débats ne prennent pas la même ampleur dans ces deux pays, notamment ceux qui ont trait en France au passé colonial. Des associations et divers mouvements agissent, en effet, de manière plus visible en France pour une reconsidération des héritages symboliques, tant sur le plan politique que sur le registre culturel, et pour une définition de la communauté nationale prenant en compte ses multiples composantes. La volonté de combattre et de dépasser les stigmatisations et l'ethnicisation, les blessures du passé ou les ambivalences relatives à l'attachement à l'État-nation dépendent évidemment de l'âge et de l'histoire familiale. Mais on observe en France que des militants ou des enfants d'immigrés s'appliquent à faire reconnaître une double appartenance pour dire le lien sensible au pays d'origine des parents et la volonté d'une citoyenneté pleine et entière en France. $\mathrm{Ce}$ processus génère des tensions et rappelle des épisodes douloureux. On le voit avec la relation à l'Algérie et les débats récurrents sur les discriminations, le racisme et encore plus sur des pratiques attachées au passé colonial, comme la torture durant la guerre de décolonisation. Bien sûr, dans ce dernier cas, les parties prenantes du débat - immigrés, harkis, rapatriés, sans oublier les historiens ou les militants anticolonialistes - prennent des positions plus ou moins divergentes, marquées par les héritages historiques et symboliques. Il serait éclairant d'approcher sous cet angle, au Japon, les reconsidérations à l'œuvre ou revendiquées, notamment pour, et éventuellement par, les populations immigrées comme les Coréens. 